\title{
Prognostic value of PPM1D in 800 gastric cancer patients
}

\author{
DAN MA, CHAO-JUN ZHANG, ZU-LIN CHEN and HUA YANG
}

Department of General Surgery, The Second Affiliated Hospital of The Third
Military Medical University, Chongqing 400037, PR China

Received July 27, 2013; Accepted March 19, 2014

DOI: $10.3892 / \mathrm{mmr} .2014 .2165$

\begin{abstract}
Protein phosphatase magnesium-dependent 1 delta (PPM1D) has recently been associated with tumor biology. However, the expression pattern and clinical significance of PPM1D in gastric cancer (GC) have yet to be elucidated. The present study aimed to investigate the clinical and prognostic significance of PPM1D in GC.PPM1D expression was assessed in 800 patients with GC using immunohistochemistry and tissue samples were divided into a PPM1D-positive and -negative group. The correlation between PPM1D expression and clinicopathological parameters or prognosis was investigated. PPM1D expression was significantly higher in GC tissue than in adjacent normal tissue (48 versus 9.5\%; $\mathrm{P}<0.001$ ). PPM1D positivity was significantly correlated with nodal status, distant metastasis and vascular invasion. Survival analysis indicated that the five-year survival rate in the PPM1D-positive group was significantly lower than that in the PPM1D-negative group (41 versus $72 \%$; $p=0.0012$ ). Furthermore, the association between PPM1D positivity and survival rate was still significant following regulation of other prognostic markers in a multivariate analysis [hazard ratio (HR), 6.572; 95\% confidence interval (CI), 3.108-13.471; $\mathrm{P}=0.0018]$. In conclusion, the present study suggested that PPM1D positivity is associated with GC invasion and metastasis, and proposed PPM1D positivity as an indicator of unfavorable prognosis in patients with GC.
\end{abstract}

\section{Introduction}

Gastric cancer (GC) is one of the most common types of malignancies and the second leading cause of cancer-associated mortalities worldwide (1). Despite rapid advances in current treatment protocols incorporating chemoradiation into surgical procedures, GC continues to be a lethal disease with the majority of patients being diagnosed at advanced stages

Correspondence to: Dr Hua Yang, Department of General Surgery, The Second Affiliated Hospital of The Third Military Medical University, 183 Shapingbaxinqiao Street, Chongqing 400037, P.R. China

E-mail: cqyanghua@yeah.net

Key words: gastric cancer, PPM1D, prognosis, biomarker at which palliative therapy is the only remaining treatment option (2). Diagnosis at early clinical stages and feasibility of curative surgery are the most important factors for the successful treatment of GC. However, nodal invasion, distant metastasis and local relapses frequently occur even following comprehensive therapy (3). Metastasis is the major cause of mortality; however, the mechanism underlying metastatic progression in GC is highly complex and remains to be fully elucidated $(4,5)$. Thus, in order to facilitate therapeutic intervention, it is important for clinicians to identify specific biomarkers for the prediction of metastatic progression and prognosis of patients with GC.

Type 2C protein phosphatase (PP2C) family proteins are known to be involved in a wide range of physiological functions including cellular stress response signaling, apoptosis and regulation of the cell cycle (6). One member of this family is the protein phosphatase magnesium-dependent 1 delta (PPM1D). Of note, PPM1D-deficient mice are more resistant to mammary tumor formation induced by the human epidermal growth factor receptor 2 (Erbb2) or Harvey rat sarcoma (Hras) oncogenes than wild-type mice with intact PPM1D (7). Mounting evidence suggests the involvement of PPM1D in tumorigenesis. PPM1D was also shown to be overexpressed in human primary breast, ovarian and neuroblastoma tumors (8-10). More importantly, PPM1D is able to complement several oncogenes, including Ras, myelocytomatosis (Myc) and Erbb2, for cellular transformation both in vitro and in vivo $(7,8,11)$.

However, the expression pattern of PPM1D and its clinical significance in GC have yet to be elucidated. The present study aimed to investigate the expression pattern and clinicopathological involvement of PPM1D in GC. The present study provided evidence that PPM1D expression was significantly upregulated in GC tissue as compared with the adjacent normal tissue and was significantly associated with metastasis and lower survival rates of patients with GC.

\section{Materials and methods}

Patients. Data were collected from 800 patients with GC who received resection at The Second Affiliated Hospital of The Third Military Medical University (Chongqing, China). The patients included 508 males and 292 females, with a mean age of 61 years (range, 40-86). None of the patients received chemotherapy prior to surgery. All the patients received a $\mathrm{R} 0$ resection above a $\mathrm{D} 1 \mathrm{lymph}$ node dissection. The present 
study was approved by the Ethics Committee of The Third Military Medical University (Chongqing, China), and informed consent was obtained from all participants.

Assessment of PPM1D expression using immunohistochemistry. Immunohistochemical analysis was performed according to the manufacturer's instructions (Dako, Carpinteria, CA, USA). Paraffin-embedded sections ( $4 \mu \mathrm{m})$ were prepared on silane-coated slides (Sigma, St. Louis, $\mathrm{MO}$, USA), deparaffinized and incubated with $3 \% \mathrm{H}_{2} \mathrm{O}_{2}$ in methanol for $10 \mathrm{~min}$ to block endogenous tissue peroxidase. The antigen was retrieved at $95^{\circ} \mathrm{C}$ for $20 \mathrm{~min}$ in $10 \mathrm{mM}$ sodium citrate buffer. The slides were then incubated with rabbit anti-PPM1D antibody (1:50, LifeSpan BioSciences, Seattle, WA, USA) at $4^{\circ} \mathrm{C}$ overnight. Following incubation with biotinylated secondary antibody for $30 \mathrm{~min}$ at room temperature, the slides were incubated with streptavidin-peroxidase complex for $30 \mathrm{~min}$ at room temperature. Immunostaining was developed by using streptavidin peroxidase 3,3'-diaminobenzidine-chromogen detection. Rabbit immunoglobulin $\mathrm{G}(\mathrm{IgG})$ isotope controls were used, which showed negative staining.

Assessment of PPM1D positivity in GC tissue samples. All the slides were evaluated by two independent investigators who were blinded to this study. Immunoreactivity was graded as: +++ (3); ++ (2); + (1) and - (0). Score 0-1 was classified as PPM1D-negative and score 2-3 as PPM1D-positive. Patients were divided into two groups according to PPM1D positivity. The correlations between clinicopathological parameters and PPM1D expression were investigated.

Western blot analysis. GC tissue samples were analyzed using a $10 \%$ polyacrylamide gel in a sodium dodecyl sulfate buffer by electrophoresis. Gels were transferred onto a nitrocellulose membrane and incubated with anti-PPM1D antibody (Abcam, Cambridge, MA, USA). Binding of PPM1D antibody was revealed by chemiluminescence following incubation with horseradish peroxidise-conjugated goat anti-mouse antibody (Bio-Rad, Hercules, CA, USA). GAPDH (Qiagen, Hilden, Germany) was used as the internal control.

Statistical analysis. Statistical analysis was performed using the $\chi^{2}$ test with SPSS 16.0 software (SPSS, Inc., Chicago, IL, USA). Cumulative survival curves were analyzed by the Kaplan-Meier method and statistical significance was calculated using the log-rank test. The Cox proportional hazard model was used in the univariate and multivariate analyses to determine prognostic factors. A difference of $\mathrm{P}<0.05$ was considered statistically significant.

\section{Results}

PPMID protein staining of GC tissue samples. Immunohistochemistry was performed on GC tissue and adjacent non-cancerous mucosa of 800 patients. PPM1D staining showed a cytoplasmic staining pattern in most of the GC tissue sections. PPM1D expression was detected in $48 \%(384 / 800)$ GC samples with moderate to strong staining (Fig. 1A) versus 9\% (72/800) in normal paired
Table I. Correlation between clinical variables and PPM1D positivity.

\begin{tabular}{lccc}
\hline & \multicolumn{2}{c}{ PPM1D positivity } & \\
\cline { 2 - 3 } Variables & Yes, n=356 & No, n=444 & P-value \\
\hline Gender & & & \\
Male & 232 & 276 & N.S. \\
Female & 124 & 168 & \\
Tumor depth & & & \\
T1+T2 & 143 & 237 & \\
T3+T4 & 213 & 207 & N.S. \\
Nodal involvement & & & \\
Yes & 154 & 164 & $<0.001$ \\
$\quad$ No & 202 & 280 & \\
Distant metastasis & & & \\
Yes & 208 & 241 & $<0.001$ \\
$\quad$ No & 148 & 203 & \\
Vascular invasion & & & \\
Yes & 191 & 165 & $<0.001$ \\
$\quad$ No & 165 & 279 & \\
Stage & & & \\
I+II & 158 & 229 & \\
III+IV & 198 & 215 & \\
Histology & & & \\
Differentiated & 156 & 202 & N.S. \\
Undifferentiated & 200 & 242 & \\
\hline
\end{tabular}

N.S., not significant.

gastric mucosa (Fig. 1B). Similar results were obtained for PPM1D expression by western blot analysis (Fig. 1C).

Clinicopathological characteristics and PPMID positivity in GC tissue samples. The correlation between PPM1D positivity and clinicopathological parameters with regard to patient prognosis was analyzed (Table I). PPM1D positivity significantly correlated with the nodal status $(\mathrm{p}<0.001)$, distant metastasis $(\mathrm{p}<0.001)$ and vascular invasion $(\mathrm{p}<0.001)$. There was no correlation between tumor histology and PPM1D expression. The five-year survival rate of the PPM1D-positive group was significantly lower than that of the PPM1D-negative group (41 versus $72 \%$; $\mathrm{p}=0.0012$ ) (Fig. 2).

Univariate and multivariate analyses. Results of the univariate analysis are shown in Table II. Overall survival was significantly correlated with the nodal status, distant metastasis, stage, vascular invasion and PPM1D positivity. Furthermore, the association between PPM1D positivity and survival was still significant following regulation of other prognostic markers in multivariate analysis [hazard ratio (HR), 6.572; 95\% confidence interval (CI), 3.10813.471; $\mathrm{P}=0.0018$ ] (Table III). In addition, multivariate analysis showed that the depth of invasion (HR, 2.121; 95\% CI, 1.081-4.243; $\mathrm{P}=0.021$ ), nodal status (HR, 3.813; 95\% CI, 
Table II. Univariate analysis of prognosis in 800 patients with gastric cancer.

\begin{tabular}{lcr}
\hline Variables & HR $(95 \%$ CI $)$ & P-value \\
\hline $\begin{array}{l}\text { Depth of invasion } \\
\text { T1+T2 }\end{array}$ & $1.021(0.401-2.021)$ & 0.079 \\
T3+T4 & $3.203(1.947-4.833)$ & $<0.001$ \\
Nodal status & & \\
N0 & & \\
N1+N2+N3 & $9.197(2.603-20.441)$ & $<0.001$ \\
Distant metastasis & & \\
Absent & & $<0.001$ \\
Present & $2.107(1.321-4.026)$ & \\
Stage & & $<0.001$ \\
I + II & & \\
III + IV & $3.112(1.667-5.394)$ & \\
Vascular invasion & & \\
Absent & & \\
Present & & \\
PPM1D & & \\
Positive & & \\
Negative & & \\
\hline
\end{tabular}

Statistical tests were two-tailed and the significance level was $\mathrm{P}<0.05$. HR, hazard ratio; $\mathrm{CI}$, confidence interval. PPM1D, protein phosphatase magnesium-dependent 1 delta.

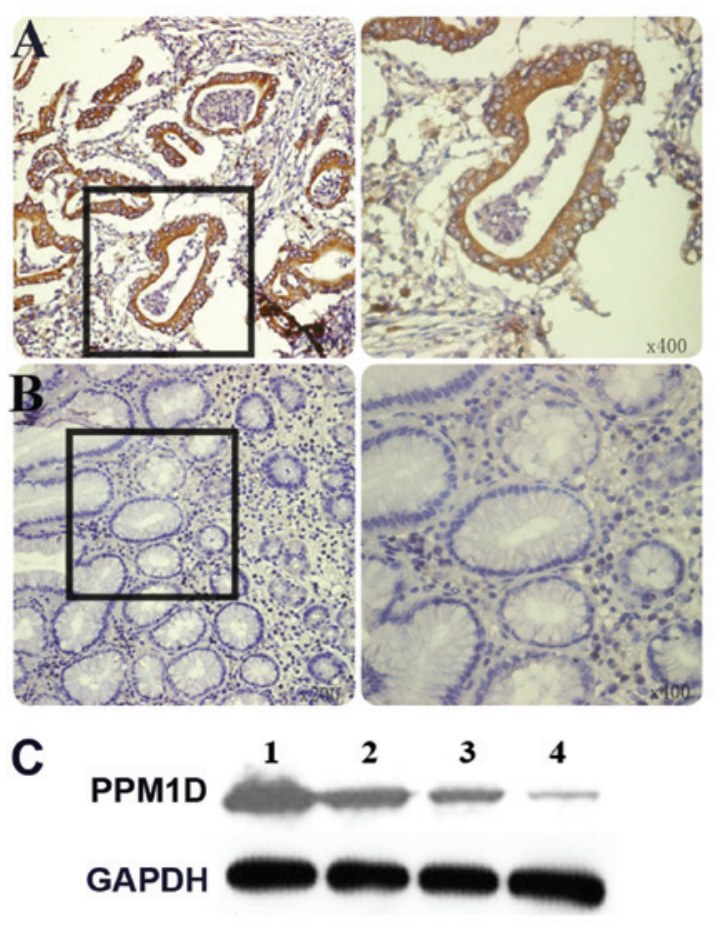

Figure 1. Expression of PPM1D in GC and adjacent normal tissue. (A) Positive PPM1D expression (GC tissue); (B) negative PPM1D expression (adjacent normal tissue); magnification, x200 (left), x400 (right). (C) Western blot analysis was performed to confirm PPM1D expression in GC tissue; lanes 1-2, gastric carcinoma, lanes 3-4, adjacent normal tissue. GC, gastric cancer. PPM1D, protein phosphatase magnesium-dependent 1 delta; GADPH, glyceraldehyde 3-phosphate dehydrogenase.
Table III. Multivariate analysis of prognosis in 800 patients with gastric cancer.

\begin{tabular}{lcc}
\hline Variables & HR $(95 \%$ CI $)$ & P-value \\
\hline $\begin{array}{l}\text { Depth of invasion } \\
\text { T1+T2 }\end{array}$ & $2.121(1.081-4.243)$ & 0.021 \\
T3+T4 & $3.813(1.526-7.907)$ & 0.013 \\
Nodal status & & \\
N0 & & \\
N1+N2+N3 & $13.701(6.091-29.772)$ & $<0.001$ \\
Distant metastasis & & \\
Absent & & \\
Present & $0.655(0.321-1.103)$ & \\
Stage & & 0.43 \\
I+II & & \\
III+IV & & \\
Vascular invasion & $1.598(1.012-3.106)$ & \\
Absent & & \\
Present & & \\
PPM1D & & \\
Positive & & \\
Negative & & \\
\hline
\end{tabular}

Statistical tests were two-tailed and the significance level was $\mathrm{P}<0.05$. HR, hazard ratio; CI, confidence interval; PPM1D, protein phosphatase magnesium-dependent 1 delta.

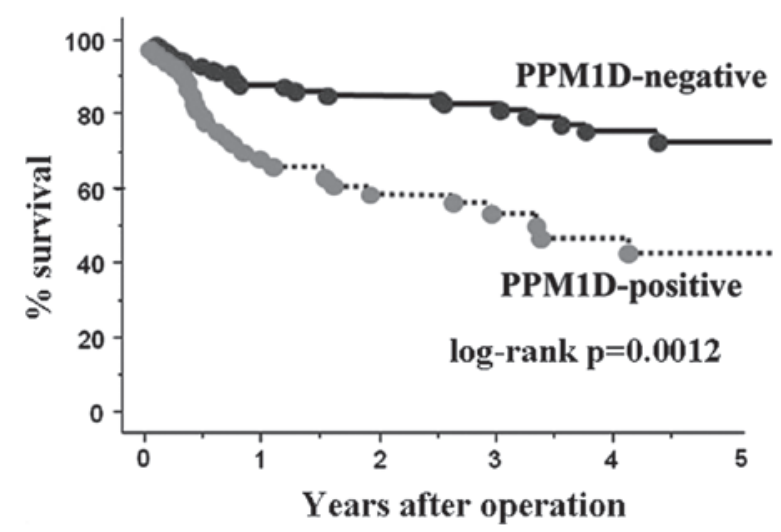

Figure 2. Survival rate of patients with gastric cancer in the PPM1D-positive group was significantly lower than that of the PPM1D-negative group (41 versus $72 \% ; \mathrm{P}=0.0012$ ). PPM1D, protein phosphatase magnesium-dependent 1 delta.

1.526-7.907; $\mathrm{P}=0.013)$, distant metastasis $(\mathrm{HR}, 13.701 ; 95 \%$ CI, 6.091-29.772; $\mathrm{P}<0.001)$ and vascular invasion (HR, 1.598; 95\% CI, 1.012-3.106; $\mathrm{P}=0.031)$ were also independent prognostic factors.

\section{Discussion}

GC-associated mortality closely correlates with early metastasis and strong invasion; accordingly, it is highly important 
to estimate the malignant degree and metastatic tendency of cancer in order to make timely and appropriate treatment decisions. The present study used immunohistochemistry to analyze the expression of PPM1D in a large group of patients with GC $(n=800)$. The results indicated that PPM1D positivity was significantly higher in GC tissue samples compared with normal gastric tissue. In addition, PPM1D positivity associated with high expression levels of PPM1D in GC lesions was significantly correlated with the nodal status, distant metastasis and vascular invasion. PPM1D upregulation was an independent prognostic factor in GC. Therefore, PPM1D may be of great value as a prognostic marker for GC.

Previous studies have indicated that PPM1D is highly expressed in neuroblastoma as well as pancreatic, lung, bladder, liver, ovarian and breast cancer $(9,10,12,13)$. However, the expression pattern of PPM1D in GC has yet to be elucidated. In the present study, immunohistochemical analysis demonstrated that PPM1D was highly expressed in GC. In addition, correlations between PPM1D expression, unfavorable prognosis and other clinical factors in GC were determined. PPM1D was upregulated in gastric cancer tissue compared with non-cancerous tissue, suggesting that PPM1D overexpression is closely associated with the progression and prognosis of GC. A number of independent studies support the pathogenic role of PPM1D in cancer biology. The overexpression of PPM1D contributes to malignant progression by inactivating wild-type p53 and p38 mitogen-activated protein kinase (MAPK) as well as decreasing p16 protein levels in human breast tissue (14). Oncogenic PPM1D is a prognostic marker for lung adenocarcinoma (15). Hu et al reported that high expression of PPM1D is closely correlated with unfavorable prognosis in pancreatic neuroendocrine tumors (16). In a follow-up study of patients with medulloblastoma, high expression levels of PPM1D were correlated with unfavorable prognosis (17). In the present study, a follow-up of patients treated for GC was performed. To the best of our knowledge, it was demonstrated for the first time that patients with GC and positive PPM1D expression had more unfavorable outcomes than those with negative PPM1D expression. This finding leads to the conclusion that PPM1D is a potential factor for outcome assessment. Furthermore, the present study has shown that the positivity for PPM1D was an independent indicator for GC. Results of the multivariate analysis revealed that PPM1D positivity, along with the depth of invasion, nodal status, distant metastasis and vascular invasion, was also an independent prognostic factor for patients with GC.

In conclusion, the present has shown showed that patients with GC overexpressed PPM1D, which was associated with aggressive clinical features and unfavorable prognosis. Therefore, PPM1D may have a significant role in the progression of GC. In addition, this information may provide novel therapeutic and prognostic possibilities for treating GC and improving the outcome of patient treatments. Thus, the roles of PPM1D in cancer are worthy of being further elucidated.

\section{Acknowledgements}

This study was supported by the National Natural Science Foundation of China (Grant no. 81071978).

\section{References}

1. Ferlay J, Shin HR, Bray F, Forman D, Mathers C and Parkin DM: Estimates of worldwide burden of cancer in 2008: GLOBOCAN 2008. Int J Cancer 127: 2893-2917, 2010.

2. Blum MA, Takashi T, Suzuki A and Ajani JA: Management of localized gastric cancer. J Surg Oncol 107: 265-270, 2013.

3. Okamoto T, Tsuburaya A, Kameda Y, et al: Prognostic value of extracapsular invasion and fibrotic focus in single lymph node metastasis of gastric cancer. Gastric Cancer 11: 160-167, 2008.

4. Zagouri F, Papadimitriou CA, Dimopoulos MA and Pectasides D: Molecularly targeted therapies in unresectable-metastatic gastric cancer: a systematic review. Cancer Treat Rev 37: 599-610, 2011.

5. Jang BG and Kim WH: Molecular pathology of gastric carcinoma. Pathobiology 78: 302-310, 2011.

6. Lu G and Wang Y: Functional diversity of mammalian type $2 \mathrm{C}$ protein phosphatase isoforms: new tales from an old family. Clin Exp Pharmacol Physiol 35: 107-112, 2008.

7. Bulavin DV, Phillips C, Nannenga B,Timofeev O, Donehower LA, Anderson CW, Appella E and Fornace AJ Jr: Inactivation of the Wip1 phosphatase inhibits mammary tumorigenesis through p38 MAPK-mediated activation of the p16(Ink4a)-p19(Arf) pathway. Nat Genet 36: 343-350, 2004.

8. Li J, Yang Y, Peng Y, Austin RJ, van Eyndhoven WG, Nguyen KC, Gabriele T, McCurrach ME, Marks JR, Hoey T, Lowe SW and Powers S: Oncogenic properties of PPM1D located within a breast cancer amplification epicenter at 17q23. Nat Genet 31: 133-134, 2002.

9. Hirasawa A, Saito-Ohara F, Inoue J, Aoki D, Susumu N, Yokoyama T, Nozawa S, Inazawa J and Imoto I: Association of 17q21-q24 gain in ovarian clear cell adenocarcinomas with poor prognosis and identification of PPM1D and APPBP2 as likely amplification targets. Clin Cancer Res 9: 1995-2004, 2003.

10. Saito-Ohara F, Imoto I, Inoue J, Hosoi H, Nakagawara A, Sugimoto T and Inazawa J: PPM1D is a potential target for $17 \mathrm{q}$ gain in neuroblastoma. Cancer Res 63: 1876-1883, 2003.

11. Demidov ON, Kek C, Shreeram S, Timofeev O, Fornace AJ, Appella E and Bulavin DV: The role of the MKK6/p38 MAPK pathway in Wip1-dependent regulation of ErbB2-driven mammary gland tumorigenesis. Oncogene 26: 2502-2506, 2007.

12. Loukopoulos P, Shibata T, Katoh H, Kokubu A, Sakamoto M, Yamazaki K, Kosuge T, Kanai Y, Hosoda F, Imoto I, Ohki M, Inazawa $\mathbf{J}$ and Hirohashi S: Genome-wide array-based comparative genomic hybridization analysis of pancreatic adenocarcinoma: identification of genetic indicators that predict patient outcome. Cancer Sci 98: 392-400, 2007.

13. Wang P, Rao J, Yang H, Zhao H and Yang L: Wip1 over-expression correlated with TP53/p14(ARF) pathway disruption in human astrocytomas. J Surg Oncol 104: 679- 684, 2011.

14. Yu E, Ahn YS, Jang SJ, Kim MJ, Yoon HS, Gong G and Choi J: Overexpression of the wip1 gene abrogates the p38 MAPK/p53/Wip1 pathway and silences p16 expression in human breast cancers. Breast Cancer Res Treat 101: 269-278, 2007.

15. Satoh N, Maniwa Y, Bermudez VP, Nishimura K, Nishio W, Yoshimura M, Okita Y, Ohbayashi C, Hurwitz J and Hayashi Y: Oncogenic phosphatase Wip1 is a novel prognostic marker for lung adenocarcinoma patient survival. Cancer Sci 102: 1101-1106, 2011.

16. Hu W, Feng Z, Modica I, Klimstra DS, Song L, Allen PJ, Brennan MF, Levine AJ and Tang LH: Gene amplifications in well-differentiated pancreatic neuroendocrine tumors inactivate the p53 pathway. Genes Cancer 1: 360-368, 2010.

17. Castellino RC, De Bortoli M, Lu X, Moon SH, Nguyen TA, Shepard MA, Rao PH, Donehower LA and Kim JY: Medulloblastomas overexpress the p53-inactivating oncogene WIP1/PPM1D. J Neurooncol 86: 245-256, 2008. 